\title{
1 Le français langue seconde, langue de la relation intime, de la relation à soi et à l'autre
}

\author{
Madeleine van Strien-Chardonneau \& \\ Marie-Christine Kok Escalle
}

Strien-Chardonneau, Madeleine van \& Marie-Christine Kok Escalle (eds.), French as Language of Intimacy in the Modern Age. Le français, langue de l'intime à l'époque moderne et contemporaine. Amsterdam: Amsterdam University Press, 2017.

DOI: 10.5117/9789462980594/CHO1

\begin{abstract}
During the Ancien Régime but also in nineteenth-century Europe, the French language, which occupied a special position in international commerce and diplomacy, develops increasingly into the language of culture and education. The language of the Republic of Letters also serves as the language of contacts and self-expression within European elites. After a rapid survey of studies devoted to this phenomenon, we concentrate on the more specific use of French as a second language in the sphere of private life. All this on the basis of a corpus of partly unpublished material written by people from the Netherlands, Italy, Russia and Turkey. These personal documents (diaries, correspondence, autobiographical texts and alba amicorum) make interesting material enabling us to reach a better understanding of the controversial question of the genesis of expressing one's feelings in texts written in the first person singular. In this context we may distinguish several functions of French. It is not only the language used by the elites but also, due to the influence of French literature, that of the heart, the language of introspection, and paradoxically that of becoming aware of one's national identity.
\end{abstract}

* Marie-Christine Kok Escalle, ICON, Institute for Cultural Inquiry, Universiteit Utrecht; Madeleine van Strien-Chardonneau, LUCAS, Leiden University Centre for Arts in Society, Universiteit Leiden 
Keywords: francophonie, French as second language, French language of private life, intimacy, personal documents, semi-public communication, semi-intimate communication

\section{Une francophonie historique}

La langue française a, dans l'Europe d'avant la Révolution française, un statut privilégié et en particulier au temps de Louis XIV, où politique, économique et culturel vont de pair pour affirmer une domination certaine. L'usage du français langue de prestige dans la circulation internationale aux dix-septième et dix-huitième siècles s'inscrit dans une histoire. Le rayonnement du français en Europe a d'abord été le fait de circonstances politiques et religieuses, comme en Angleterre où le roi de France était le suzerain du roi d'Angleterre ou aux Pays-Bas à la cour de Bourgogne, puis dans les nouvelles Provinces-Unies qui accueillent les réfugiés protestants francophones, wallons au seizième, huguenots au dix-septième siècle. À l'époque moderne, le latin qui joue le rôle de lingua franca va céder la place aux langues vernaculaires, l'italien d'abord porteur d'Humanisme, de Renaissance et de Baroque, puis le français qui de langue de 'commodité' va devenir langue de culture, langue d'éducation et langue de la République des lettres. ${ }^{1}$ L'usage du français dans la sphère publique ne se limite pas aux relations commerciales et diplomatiques, aux échanges dans les cours européennes ou encore dans le monde savant pour la diffusion des sciences, le français devient une langue de distinction, ${ }^{2}$ politique et sociale, dans l'Europe francisée du dix-huitième siècle et dans l'Europe élargie au dix-neuvième mais aussi une langue que s'approprient les élites européennes pour s'exprimer entre elles.

Ce phénomène a été étudié par Ferdinand Brunot qui a publié au début du vingtième siècle la monumentale Histoire de la langue française des origines à 1900, 'dix mille pages grand format étalées de 1905 à sa mort en 1938, et au-delà, jusqu'en 1979, vingt fascicules, onze tomes!',3 dont Pierre Nora a fait un lieu de mémoire. Les volumes 5 et 8 traitent du français hors de France aux dix-septième et dix-huitième siècles, publiés l'un en 1917, l'autre en 1934-35, dans un moment de tension avec l'Allemagne. Brunot étudie l'expansion du français en Europe, due au prestige de la culture française 
dominante; puis il suit pas à pas le retournement qui s'opère au moment de la Révolution française et de l'Empire de Napoléon $I^{\text {er: }}$ alors que la langue française est pour la révolution l'instrument de la libération des peuples, les langues locales s'affirmant contre le français vont alimenter les débuts du nationalisme qui va se développer au dix-neuvième siècle. ${ }^{4}$ Brunot traite du français en Angleterre, aux Pays-Bas et en Allemagne au dix-septième siècle et au dix-huitième siècle dans l'Europe du Nord, mais aussi, bien que de façon beaucoup plus modeste, en Italie, Espagne et Portugal.

Avec Brunot comme modèle, considéré par André Reboullet comme 'père fondateur', le premier conseil étant 'Lisez Brunot, relisez le sans cesse!', la SIHFLES 5 publie des études sur l'histoire de la présence et de l'usage du français langue étrangère ou seconde hors de France et en particulier sur l'histoire de son enseignement. L'approche pluri- et interdisciplinaire que prône la SIHFLES intéresse littéraires, linguistes, sociologues, sociolinguistes, historiens de la culture et de la langue, travaillant sur des sources très diverses et dans de nombreux pays, surtout européens. Les parcours et les pratiques des maîtres, les contenus des manuels utilisés pour l'apprentissage du français, langue étrangère et/ou seconde, les programmes d'instruction et d'éducation en français, font l'objet des recherches dans les trois dimensions - linguistique, sociologique et pédagogique - avancées par Brunot. ${ }^{6}$ L'usage du français hors d'un contexte à dominante francophone étant une pratique culturelle, il faut la situer par rapport à l'appropriation ou non de modèles culturels, par rapport à des facteurs contextuels, externes à la langue, comme le facteur religieux ou politique par exemple. Si les écrits de la sphère privée ont peu fait l'objet d'études dans les publications SIHFLES, on peut toutefois mentionner l'intérêt porté aux correspondances des précepteurs francophones chargés d'éduquer en français en Europe ${ }^{7}$ ainsi qu'à celle des correspondants suisses du secrétaire de l'Académie de Berlin (Formey 1711-1797). ${ }^{8}$

Dans la lignée en quelque sorte de Brunot paraît, en 2007, Mille ans de langue française. Histoire d'une passion, 'ouvrage [qui] a pour ambition de montrer les origines et les usages du français tel qu'il est parlé et écrit en

4 Chevalier, 'Ferdinand Brunot et le français langue étrangère', 12-14.

5 La Société Internationale pour l'Histoire du Français Langue Étrangère ou Seconde, fondée en 1987 publie Documents pour l'histoire du français langue étrangère ou seconde $(1,1988,54$ et 55, 2015). En outre, deux numéros de Recherches et Applications. Le français dans le monde ont proposé deux synthèses à quinze ans d'intervalle (1998, 2012).

6 Minerva, 'De Ferdinand Brunot à la SIHFLES', 180-186.

7 Rjéoutski, Le précepteur francophone en Europe (XVII $-X I X^{e}$ siècles).

8 Bandelier, 'Échanges épistolaires et préceptorat des Lumières', 145-173.

\section{Amsterdam University Press}


France, mais aussi à travers le monde'. ${ }^{9}$ Dans la préface, l'un des coauteurs, Gilles Siouffi, en précise l'objectif: 'raconter cette longue histoire de la langue française [...] envisagée [...] dans toute sa diversité et sa complexité sociale, culturelle et anthropologique'. ${ }^{10}$ Louvrage offre également, sous forme de panorama succinct, deux chapitres sur le français hors de France, respectivement au dix-septième siècle $\left(\mathrm{I}, 5^{81-606)}\right.$ et au dix-huitième siècle (II, 39-70) avec des synthèses concernant divers pays européens, Angleterre, Allemagne, Pays-Bas (Hollande et Belgique), Italie, Portugal, Espagne. Ces courtes synthèses, certes très utiles, ont parfois l'inconvénient d'être un peu rapides: ainsi, si l'on souligne à juste titre le rôle majeur des Pays-Bas 'dans la diffusion en Europe du français' (I, 582 ), on évoque un peu trop rapidement le 'désamour' du français en Hollande au dix-huitième siècle (II, 52-53). Les sentiments gallophobes sont assurément bien présents, mais on ne dédaigne pas de les exprimer en français, ${ }^{11}$ langue qui, en dépit de la montée en puissance incontestable de l'allemand et de l'anglais, reste encore pratiquée jusque dans la première moitié du vingtième siècle.

Lors du colloque international organisé en 2011 par Elena Gretchanaia, Alexandre Stroev et Catherine Viollet, 'La francophonie européenne aux XVIII - XIX ${ }^{\mathrm{e}}$ siècles' et dans l'ouvrage au même titre paru en 2012, les organisateurs ambitionnent de 'constituer la francophonie européenne des $\mathrm{XVIII}^{\mathrm{e}}-\mathrm{XX}{ }^{\mathrm{e}}$ siècles en tant que nouveau domaine de recherche ${ }^{12}$ et proposent tout d'abord une réflexion sur la notion de francophonie. Dans la première contribution, Jean Bessière remarque: 'Les études littéraires francophones se sont constituées, on le sait, pour l'essentiel en prenant pour occasion, pour support et pour objet, les littératures des pays qui ont appartenu à l'Empire français et qui sont devenus indépendants. ${ }^{13}$ Cette dimension de la francophonie est en effet toujours très présente, par exemple, dans les congrès internationaux organisés annuellement par le CIEF. ${ }^{14}$ Bessière souligne également que cette perspective des études littéraires francophones ignore la diversité des francophonies hors des anciens pays colonisés, et c'est

9 Rey, Duval \& Siouffi, Mille ans de langue française, $4^{\mathrm{e}}$ de couverture de l'édition revue et augmentée, 2011.

10 Rey, Duval \& Siouffi, Mille ans de langue française, 7 .

11 Ce phénomène n'est pas propre aux Pays-Bas (voir Argent, Rjéoutski \& Offord, 'European Francophonie and a Framework for Its Study' in European Francophonie, 17: 'Siouffi regards the highly developed Gallophobia that can be observed at certain times in Italy, Spain and England as proof that francophonie was not widespread in those lands, but the chapters in this volume on Italy and Spain do not entirely bear out this point of view').

12 Gretchanaia, Stroev \& Viollet, La francophonie européenne aux XVIII ${ }^{e}$-XIX ${ }^{e}$ siècles, 13.

13 Ibidem, 29.

14 Conseil international des Études francophones, http://www.cief.org. 
précisément ce vide que La francophonie européenne entend combler, en examinant dans un contexte historique les auteurs d'expression française des pays de l'Europe de l'Est, de l'Europe centrale et de l'Europe du Nord, avec un intérêt particulier pour la Russie.

Dans European Francophonie, volume lui aussi édité par des spécialistes du monde russe, deux ans après le précédent, les éditeurs reprennent, en la précisant, la distinction entre, d'une part 'francophonie moderne', référant aux études évoquées ci-dessus par Bessière, et d'autre part 'francophonie européenne ou historique.. ${ }^{15}$ Ils rappellent l'intérêt assez récent des chercheurs pour la diffusion du français langue seconde en Europe, à partir du dix-septième siècle, ce champ constituant l'objet du volume, spécifiant bien qu'ils n'y incluent pas des communautés originellement francophones telles qu'on les trouve en Belgique ou en Suisse. ${ }^{16}$ Ce volume répond donc dans une certaine mesure au souhait exprimé par les éditeurs de La francophonie européenne... qui présentaient leur ouvrage comme une première étape. Le champ étudié s'est en effet considérablement élargi aussi bien chronologiquement que géographiquement puisque les 12 chapitres du volume European Francophonie... couvrent une période allant du milieu du dix-septième siècle (avec une incursion dans le Moyen-Âge anglais) jusqu'au début du vingtième siècle et embrassent l'histoire du français langue seconde dans 12 pays différents (Angleterre, Piémont, Italie, Pays-Bas (du Nord), Prusse, Bohême, Espagne, Pologne, Suède, Roumanie, Russie, Turquie).

Deux volumes publiés par le groupe de recherche dirigé par Derek Offord explorent en détail la position du français et sa relation avec le russe dans la Russie impériale. ${ }^{17}$ Ses travaux se poursuivent dans le but de publications ultérieures, en étudiant en particulier de nombreux documents à la frontière entre le document privé et le document public, journaux et lettres écrits en français au sein de la noblesse russe à la fin du dix-huitième siècle et au dix-neuvième. Le cas russe et les études qu'il a suscitées sont particulièrement intéressants dans leur effort comparatif qui, s'il permet de valider les observations sur les pratiques du français en Russie, inscrit aussi ces mêmes pratiques dans une perspective européenne et offre aux chercheurs d'autres pays de très utiles éléments de comparaison. De plus, l'étude couplée de l'usage du français et du russe est également une démarche utile pour

15 “"Modern francophonie" and "European" or "historical francophonie", European Francophonie, 4-10.

16 'Note that we do not use the term 'European Francophonie' to refer to European speech communities that are francophone today, such as Belgium or Switzerland', ibidem, 4, note 2.

17 Offord et al., French and Russian in Imperial Russia.

\section{Amsterdam University Press}


d'autres aires linguistiques: en s'intéressant aux rapports entretenus entre le français langue seconde et la langue vernaculaire des pays étudiés, il est possible de préciser et nuancer le degré de francisation de certains groupes sociaux.

\section{De l'écriture à la première personne à l'expression de l'intime}

Sous-jacente à ces études publiées récemment ou il y a presque un siècle, la question de l'usage de la langue française, langue seconde ou étrangère comme langue d'écriture se pose. À différentes époques et dans différents pays d'Europe, les élites bourgeoises, patriciennes et aristocratiques, écrivent en français, leurs correspondances, leurs journaux de voyage, leurs journaux personnels, leurs Mémoires ou récits de vie; à ces documents qui relèvent de la sphère privée ou semi-privée, on peut ajouter des poésies de circonstance et des alba amicorum.

$\mathrm{Si}$, dans Mille ans de langue française, on minimise l'importance du français comme langue de la diplomatie, on souligne en revanche que son usage pour la correspondance privée se répandit rapidement. Outre qu'il est vu comme un bon médium pour la communication d'informations et de points de vue, le français est en quelque sorte un terrain 'neutre' qui permet de se faire autre dans l'échange, dans l'écriture, et de gommer également les différences nationales. ${ }^{18}$ Ici, comme pour la plupart des personnages que Marc Fumaroli présente dans son célèbre ouvrage Quand l'Europe parlait français, le français est utilisé entre locuteurs de langues maternelles différentes. Or, au dix-septième, dix-huitième, dix-neuvième et même encore au vingtième siècle, c'est en français que sont rédigées certaines correspondances personnelles entre les membres d'une même famille ou entre amis, qui partagent la même langue maternelle. Peut-on parler dans ce cas de 'gommage' des différences nationales ou même de terrain 'neutre'? Pourquoi s'écrit-on en français entre Néerlandais, entre Russes ou entre Turcs? Et on peut se poser la même question à propos de journaux personnels, de relations de voyage, de poésies de circonstance, d'alba amicorum mentionnés plus haut, qui circulent souvent dans le cercle familial ou amical.

18 Mille ans de langue française, histoire d'une passion, I, Des origines au français moderne, 593-6o6. Isabelle de Charrière offre un bel exemple de ce 'gommage' des différences nationales lorsqu'elle rappelle non sans nostalgie à Benjamin Constant: 'Vous et moi, nous n'étions d'aucun pays quand nous étions ensemble' (O.C., V, 26-27).

\section{Amsterdam University Press}


La contribution, dans La francophonie européenne... de Catherine Viollet, ${ }^{19}$ à qui nous rendons ici hommage, a été notre première source d'inspiration. Viollet fait ressortir, à côté de la vaste production de textes publiés en français hors de France, l'intérêt présenté, en ce qui concerne la pratique de cette langue, par des écrits moins visibles parce que restés pour la plupart inédits, mais qui n'en sont pas moins significatifs pour l'étude de la pratique du français langue seconde: ceux, mentionnés plus haut, qui relèvent de l'espace autobiographique et qui sont, pour la plus grande partie, conservés dans les archives publiques et privées. ${ }^{20} S^{\prime}$ intéressant plus spécialement aux journaux personnels, elle signale leur présence dans divers pays (outre la Russie, Pologne, République tchèque, Pays-Bas, Grande-Bretagne, Allemagne, Suède) et souligne leur intérêt comme 'documents précieux d'histoire culturelle et de microhistoire. ${ }^{21}$ Les publications résultant des travaux du groupe de recherche de Derek Offord explorent également ce champ des documents personnels. Ce dernier avait déjà souligné dans 'Francophonie in Imperial Russia ${ }^{22}$ que le français était en Russie la langue de prédilection pour les journaux de voyage et journaux personnels ainsi que celle de la correspondance privée aristocratique, non seulement pour l'échange avec des correspondants étrangers ne maitrisant pas le russe mais aussi avec des compatriotes, parents, amis, patrons et protégés. Cet aspect de l'usage du français par les élites russes est développé dans le premier tome de French and Russian in Imperial Russia. ${ }^{23}$ Plusieurs contributions portent sur des documents personnels et notent l'utilisation fréquente du français pour l'expression de l'intime. ${ }^{24}$

Il serait hasardeux de généraliser les constatations faites à propos de cas individuels mais l'étude de nombre de ces cas dans des aires linguistiques différentes, et sur une période longue, peut permettre de dégager dans ce type de documents s'inscrivant dans l'espace autobiographique, certaines

19 Viollet, 'Écrits personnels en français: une dimension européenne (fin XVIII ${ }^{\mathrm{e}}$-début XIX siècle)', in La francophonie européenne..., 37-50. Quelques articles du volume traitent de documents personnels écrits en français.

20 Dans la conclusion de l'ouvrage European francophonie, on note aussi l'intérêt que présentent ces documents personnels en français: 'Extant samples of egowriting provide invaluable material for historical sociolinguistics studies, as is shown by the significant amount of scholarly literature in this area', en citant, à l'appui, Stephan Elspass, 'The Use of Private Letters and Diaries in Sociolinguistic Investigation', in The Handboek of Historical Sociolinguistics ed. by Juan Manuel Hernandez \& Juan Camilo Conde-Silvestre (London, Blackwell, 2012), 156-169.

21 Gretchanaia \& Viollet, Si tu lis jamais ce journal..., 43.

22 Rjéoutski, Argent \& Offord, European Francophonie, 385-386.

23 Offord et al., Language use among the Russian Elite.

24 Cf. entre autres Sapchenko, 'French and Russian in Ego-Documenten', 157-159; Dulac, 'The Use of French by Catherine II', 45-6o.

\section{Amsterdam University Press}


constantes ou au contraire des différences notoires dans l'usage et les fonctions du français langue seconde.

\section{'Egodocuments': entre sphère privée et sphère publique}

Certes, il faut tenir compte du fait que la pratique de la correspondance, la tenue d'un journal de voyage ou d'un journal intime, qu'ils soient rédigés dans la langue vernaculaire ou en français langue seconde, sont aussi des pratiques de socialisation, comme l'indiquent à juste titre, pour le contexte néerlandais, Willemijn Ruberg à propos des correspondances, ${ }^{25}$ et Arianne Baggerman et Rudolf Dekker en ce qui concerne la tenue d'un journal. Ces pratiques sociales ont cependant favorisé l'écriture de soi. Ainsi Ruberg souligne le rôle des parents ou éducateurs éclairés qui proposent aux enfants de suivre des modèles au style naturel et individuel comme les lettres de Madame de Sévigné ou Lady Montagu, alliant le souci de développer la confiance et l'individualité de l'enfant à celui de faire de lui un individu policé au comportement adapté aux exigences de son appartenance sociale. ${ }^{26}$ Baggerman et Dekker, de leur côté, montrent que, vers 1750, un nombre croissant de parents encouragent leurs enfants à tenir un journal, destiné à stimuler la réflexion personnelle et à acquérir une meilleure maîtrise du temps, et que cette innovation a stimulé l'essor de l'écriture autobiographique. ${ }^{27}$

La distinction entre sphère publique et sphère privée n'est pas absolument stricte et la notion d'intime n'est pas non plus toujours nettement définie. Ruberg souligne ce point en ce qui concerne les correspondances:

The correspondence analyzed could be described as 'private' since it does not primarly concern business affairs. However, it must be taken into account that public and private sphere were not completely separated in the $18^{\text {th }}$ and $19^{\text {th }}$ centuries. [...] Letters were frequently read aloud in front of relatives.'. ${ }^{28}$

Pour Pierre-Jean Dufief également, la lettre appartient à la catégorie des écrits 'pour autrui' et elle possède par nature une dimension de

Ruberg, 'Letter Writing...', 249-251.

Ruberg, 'Children's correspondence...', 306-308.

Baggerman \& Dekker, Child of the Enlightenment, 87-91.

Ruberg, 'Letter writing...', 250.

\section{Amsterdam University Press}


communication sociale. ${ }^{29}$ Par contre, il estime que l'écriture du journal relèverait sans ambiguïté du registre de l'intime. ${ }^{30}$ Cependant, comme la lettre, le journal, nous l'avons déjà mentionné, peut être aussi destiné à une lecture extérieure, celle par exemple du précepteur, du maître ou des parents dans le cas du journal d'éducation. Les journaux de voyage des jeunes gens faisant leur Grand Tour sont également lus (comme d'ailleurs leurs lettres destinées aux parents) par le gouverneur qui évalue la qualité d'expression du français langue étrangère. Pour les voyageurs plus âgés, il est fréquent de faire circuler le journal de voyage dans le cercle d'amis. Par ailleurs, comme le fait remarquer Emilie Murphy, à propos de récits de voyage rédigés en français par des femmes russes pour la période 1777-1850, les contraintes sociales peuvent brider l'expression personnelle: 'Alors que ces récits de voyage sont des actes de l'expression de soi, une place privilégiée n'est accordée au 'je' que dans les limites des discours contemporains de la féminité. ${ }^{31}$

De plus, la frontière entre journal personnel dans lequel s'expriment les sentiments et les impressions du diariste, et le journal de voyage, plus factuel, n'est pas étanche: un journal de voyage peut parfaitement, au sein d'un même cahier, se transformer en journal personnel et l'inverse est également possible.

\section{Naissance de l'intime}

Il est indéniable que dans ce genre de textes, l'écriture à la première personne favorise aussi l'écriture de soi et l'éclosion du sentiment de l'intime. La question de l'intime est controversée puisque que pour certains, l'intimité aurait été inventée au Siècle des Lumières, cette époque 'mettant à jour un moi qui se perçoit, se formule et se manifeste selon de nouvelles modalités, définissant ce faisant, ses propres modes d'expression'. Le journal et la lettre sont considérés comme les genres privilégiés pour ces 'épanchements du ${\text { moi' } 3^{2}}^{2}$

En ce qui concerne l'apparition du terme même, en France, le nouveau Petit Robert de la langue française (2010) signale les premières occurrences dès le quatorzième siècle (en 1377 et 1390), mais pour 'intimité', une occurrence

Dufief, Les écritures de l'intime, 8.

Ibidem.

Murphy, 'Récits de voyage' in La francophonie européenne..., 227.

Cots Vicente, compte rendu de L'invention de l'intimité au siècle des Lumières. 
nettement plus tardive (1684). Dans le riche recueil d'études rassemblées par Anne Coudreuse et Françoise Simonet-Tenant sur l'histoire de l'intime, ${ }^{33}$ Véronique Montémont analyse l'évolution lexicographique du terme 'intime' entre $1606^{34}$ et 2008 et souligne que le dix-huitième siècle

constitue une sorte de transition sémantique: tout en maintenant au premier plan la problématique relationnelle, les lexicographes introduisent discrètement l'idée d'une intériorité du sujet et de sa conscience [...] C'est plus particulièrement à partir de 1760 que l'adjectif intime qui désignait jusqu'alors une relation privilégiée avec autrui - se met à impliquer un sentiment intérieur, une conscience propre au sujet. ${ }^{35}$

Simonet-Tenant considère, elle aussi, le dix-huitième siècle comme une époque charnière dans l'élaboration d'une culture de l'intimité. Abordant l'évolution des lettres et des journaux, elle met en évidence la lente montée en puissance de l'intime vers la fin de l'Ancien Régime:

Un grand mouvement vers l'intimité de l'écriture anime les scripteurs européens dans la seconde moitié du dix-huitième siècle, qui s'empare des journaux personnels et de lettres au point de faire du journal, quelques décennies plus tard, l'archétype de la littérature intime. ${ }^{3}$

Pour d'autres chercheurs, comme Georges Gusdorf, les écritures du moi sont bien plus anciennes et il signale les 7 volumes de la Geschichte der Autobiographie (1907) de Georg Misch qui développe l'histoire de l'autobiographie antique et médiévale..$^{37}$ Dans son chapitre consacré à Montaigne, Gusdorf considère les Essais (1580-1595) comme 'un journal intime, journal sans date, mais portrait au fil du temps' et il récuse la phrase liminaire du livre I des Confessions dans laquelle Rousseau affirme: 'Je forme une entreprise qui n'eut jamais d'exemple', arguant que 'lentreprise de la connaissance de soi avait tenté avant lui, toutes sortes d'esprits, petits ou grands' ${ }^{3}{ }^{8}$

Lintérêt pour les écrits du for privé, accru ces dernières années, a stimulé la création d'équipes de recherche en France, en Italie, en Suisse, en Allemagne

Coudreuse \& Simonet-Tenant, Pour une histoire de l'intime et de ses variations. 
et aux Pays-Bas. ${ }^{39}$ Celles-ci ont répertorié de nombreux documents ignorés jusqu'alors, révélant une très grande variété d'écritures autobiographiques ${ }^{40}$ bien avant le dix-huitième siècle et permettant ainsi de situer les textes publiés et bien connus dans un contexte plus large. En France, Philippe Lejeune, dont les travaux ont joué et jouent encore un rôle primordial dans l'intérêt croissant pour ce type d'écrits, rappelle, dans une conférence faite à Genève en 2012, ${ }^{41}$ les critiques formulées à son égard pour 'avoir posé Rousseau, comme 'origine' de l'autobiographie moderne'. ${ }^{42}$ Reconnaissant que Rousseau n'a rien inventé et s'inscrit dans diverses traditions, telle que l'autobiographie religieuse (Saint Augustin), l'autoportrait (Montaigne), il souligne néanmoins qu'il a 'tout reconfiguré' et que la première partie des Confessions publiée en 1782 a constitué un véritable événement. ${ }^{43}$

Rousseau, en effet, sans être absolument le fondateur d'un genre - la tradition religieuse de l'examen de conscience et de l'aveu a suscité nombre d'écrits personnels en particulier dans les milieux protestants européens ${ }^{44}$-illustre avec ses Confessions, la laïcisation du modèle de l'autobiographie spirituelle'. Il ouvre la voie à la promotion de l'intime en lui conférant 'une publicité spectaculaire en même temps que ses lettres de noblesse en littérature'. ${ }^{45}$

Ce succès dépasse les frontières. Gretchanaia note la floraison 'des écrits autobiographiques (Mémoires, journaux de voyage, journaux personnels) russes au dix-neuvième siècle, masculins et féminins, et souligne l'influence des Confessions. ${ }^{46}$ Plusieurs études de ce volume la signalent également dans des correspondances entre Néerlandais au dix-huitième siècle, ${ }^{47}$ ou entre Turcs au début du vingtième siècle..$^{8}$

39 Groupe de recherches Paris-Sorbonne, Jean-Pierre Bardet \& François Joseph Ruggieri, 'Présentation', www.ecritsduforprive.fr (consulté le 10-10-2015).

40 Voir par exemple Baggerman, Dekker \& Mascuch, Controlling Time and Shaping the Self dont les diverses contributions témoignent de l'étonnante variété des écrits autobiographiques après 1500 dans divers pays d'Europe, du Moyen-Orient et du Japon.

41 Lejeune, Conférence aux journées de l'Autobiographie (APA).

42 Voir en particulier la critique de Gusdorf sur son premier ouvrage, L'Autobiographie en France (1971) dans Les écritures du moi, 53.

43 Ibidem.

44 Lejeune note, comme Gusdorf avant lui, le développement des journaux personnels puritains dans l'Angleterre du dix-septième siècle et des journaux piétistes dans l'Allemagne du dix-huitième, mais signale la difficulté de trouver au cours de ces deux siècles un journal spirituel écrit en langue française (il précise cependant 'journal publié'). 'Les journaux spirituels', 63, 68, 75 .

45 Simonet-Tenant, 'À la recherche des prémices d'une culture de l'intime', 41.

46 Gretchanaia, 'Les écrits autobiographiques des femmes russes du XVIII ${ }^{\mathrm{e}}$ siècle rédigés en français'.

47 Cf. Van Strien-Chardonneau dans ce volume.

48 Cf. Güven \& Güven dans ce volume.

\section{Amsterdam University Press}


Plus généralement, la littérature française du siècle classique et du siècle des Lumières a offert un large éventail de genres, théâtre, fables, contes, satires, romans, essais qui ont fourni des matériaux pour l'expression des sentiments et des idées et des modèles à nombre d'auteurs européens. ${ }^{49}$ On pourrait aussi avancer, en tenant compte de l'hypothèse de Fumaroli selon laquelle le français serait 'une langue qui excellait [au XVIII ${ }^{\mathrm{e}}$ siècle] notamment dans les genres intimes, la lettre, le journal, la poésie de circonstance, les Mémoires'50, que les modèles fournis par les Mémoires, les récits de voyage et les romans épistolaires français qui connaissent de fort succès éditoriaux ont contribué à favoriser l'écriture de soi en français, langue seconde ou étrangère, non seulement chez des auteurs connus et reconnus mais encore chez ceux dont les écrits dorment dans les archives. Ces scripteurs 'ordinaires' s'approprient par le biais de l'écriture en français des moyens d'expression, entrant en dialogue avec une culture qu'ils endossent ou remettent parfois aussi en question.

\section{Les pratiques et les fonctions d'usage (semi) privé du français langue étrangère ou seconde}

Le français, 'langue en partage', comme l'affirme l'organisation politique de la Francophonie aujourd'hui, lingua franca partagée pour les échanges internationaux politiques, diplomatiques, littéraires, savants dans l'histoire, a aussi été partagé dans le domaine privé, par un groupe social restreint, constituant un bien commun, à la fois linguistique et culturel. L'usage (semi) privé du français langue étrangère ou seconde en fait une langue de distinction et d'appartenance à une élite, une langue de cœur inspirée par la littérature, la langue de la réflexion intérieure et la langue de la formation de la pensée, enfin la langue de la prise de conscience identitaire nationale.

\section{Langue de distinction des élites}

Comme on peut le voir dans les Pays-Bas, dès le seizième siècle, l'usage restreint du français dans les alba amicorum de jeunes filles nobles, présentés dans ce volume par Sophie Reinders, dénote un signe de distinction propre à un réseau relationnel spécifique. On ne peut certes parler d'expression de l'intime dans les textes conventionnels, devises ou proverbes, poèmes, 
chansons qui y sont inscrits mais l'utilisation de ces textes en français dans des 'cahiers' privés est révélatrice de ce bien commun aux membres d'un groupe social pour communiquer dans un cercle restreint et exprimer une certaine image des femmes censées répondre aux modèles proposés, qui n'est pas sans rappeler ce que l'on trouve dans les manuels de l'époque destinés à l'apprentissage du français, préceptes religieux, éloge de la vertu féminine, dangers de l'amour..$^{51} C^{\prime}$ est surtout à partir du dix-septième siècle avec le rayonnement de la société de cour ritualisée à Versailles, puis au dix-huitième, avec l'influence des philosophes des Lumières que le prestige du français s'accroît. Langue des cours européennes, le français, fonctionnant donc comme une lingua franca à laquelle on attribue certaines qualités de concision, naturel et clarté qui justifieraient son 'universalité', ${ }^{52}$ devient aussi un signe de reconnaissance pour les cercles aristocratiques..$^{53}$

L'utilisation du français dans des documents ressortissant à l'espace autobiographique est certes commandée par ce facteur de marque distinctive. Les Mémoires princiers ou aristocratiques en français, comme ceux de Catherine II, de la Margrave de Bayreuth ${ }^{54}$ ou d'Ekaterina Romanovna Vorontzoff, princesse Dashkova, ${ }^{55}$ donnent le ton, ainsi que la pratique des correspondances familiales dans le cercle des stathouders des Provinces-Unies, des cours royales et impériales de Suède, de Prusse, d'Autriche, et de Russie.

Ainsi dans les Provinces-Unies du début du dix-huitième siècle, les jeunes aristocrates frisons Willem et Onno Zwier van Haren reçoivent une éducation bilingue: le néerlandais est nécessaire à une carrière militaire et administrative, le français langue de la cour stathoudérienne, donne accès aux plus hautes sphères de la société. Carolina van Haren, la fille d'Onno, restera fidèle à la tradition de la correspondance familiale en français et rappellera à son fils Gijsbert Karel van Hogendorp que la connaissance du français, langue de la conversation et de la correspondance, est indispensable pour un homme de son rang. ${ }^{6}$ Pour Vincent van Tuyll van Serooskerken, qui avait prié sa sœur

51 Kok Escalle \& Van Strien-Chardonneau, 'Moyens linguistiques et construction identitaire sexuée', 228-235.

52 Pour la discussion de ce concept d'universalité de la langue française, voir Siouffi, 'De l'universalité' européenne du français au XVIII siècle: retour sur les représentations et les réalités', et Böhm, 'The Domains of Francophonie and Language Ideology in Eighteenth- and Nineteenth-Century Prussia', 178-185.

53 Rey, Duval \& Siouffi, Mille ans de langue française, 594.

54 Fumaroli, Quand l'Europe parlait français, 136-137.

55 Lamarche Marrese, 'Princess Dashkova and the Politics of Language in Eighteenth-Century Russia'.

56 Beaufort, Karel van Hogendorp, 37.

\section{Amsterdam University Press}


Isabelle de Charrière de s'occuper de l'éducation de son fils aîné, Willem René, il va de soi qu'un 'Hollandais doit ou devrait connaître également sa propre langue et la française'.57 Dans les deux cas, la maîtrise du français est considérée comme une marque distinctive de l'appartenance sociale.

En Bohême, les aristocrates, qui écrivent en français leurs correspondances privées et leurs journaux personnels, utilisent l'allemand dans l'administration et le service public, mais la maîtrise du français permet de délimiter la frontière entre ce groupe et le reste de la société et est le gage de l'acceptation dans ce milieu.$^{8}$ Le passage du russe au français dans la correspondance de Nikolai Karamzin avec le tsar Alexandre $\mathrm{I}^{\text {er }}$ traduit, selon Sapchenko, la volonté de diminuer la distance entre souverain et sujet, se plaçant ainsi en tant que destinateur sur le même pied que son impérial destinataire, au sein d'une même élite. ${ }^{59}$

\section{Langue du cœur inspirée par la littérature}

Si l'écriture épistolaire, (de même que l'écriture diaristique) est liée à un désir d'acquérir une compétence linguistique et culturelle, ceux qui utilisent le français langue seconde dans leur correspondance exploitent aussi, comme d'ailleurs les scripteurs de langue maternelle française, les moyens d'expression de la relation privilégiée avec autrui, avec l'ami, avec les parents, les frères et sœurs, avec la femme ou l'homme aimé(e).

Bien que tous deux de langue maternelle allemande, Melchior Grimm et l'impératrice Catherine II correspondent en français, créant ainsi une complicité qui restitue l'atmosphère amicale et intime de leurs conversations. ${ }^{60}$ La lettre n'est-elle pas une conversation par écrit, une transposition de ce genre littéraire oral qu'est la conversation entre amis et dans lequel le français et les Français avaient la réputation d'exceller ${ }^{21}$ Personnages bien plus obscurs que Grimm et Catherine II, l'homme de lettres néerlandais Onno Zwier van Haren et son ami, l'érudit Gerard Nicolaas Heerkens correspondent non pas dans leur langue maternelle, mais en français, faisant leur cette définition de la lettre, conversation entre absents ${ }^{62}$ et s'appropriant

62 'Je suis charmé que mes épîtres ne vous ennuient point, elles vont comme ma plume et mon imagination, souvent par sauts et par bonds: une lettre étant une conversation entre des 
ainsi le modèle de civilité à la française tout en discutant littérature et parlant librement de leur santé et de leurs amours.

Le français est utilisé également dans la correspondance entre parents et enfants, dans le but premier de perfectionner les compétences linguistiques de l'élève. Nombre de manuels destinés à l'apprentissage du français langue étrangère proposent des modèles de lettres. Le Suédois Carl Gustav Tessin reconnaît d'ailleurs ingénument: 'Pourquoi est-ce que je n'écris pas toutes mes drogues en suédois? C'est que j'ai plus de modèles en français' ${ }^{\prime 63}$. On recommande aussi aux épistoliers en herbe, la lecture de bons auteurs. Ainsi les lettres de Madame de Sévigné, modèles de la relation maternelle affective, jouissent d'un grand succès à partir de leur publication au cours du dix-huitième siècle. Ces lettres exercent une séduction indéniable: Catherine II avoue les avoir 'dévorées', ${ }^{64}$ la Néerlandaise Magdalena van Schinne ${ }^{65}$ compare volontiers son propre style avec celui de l'épistolière. Le précepteur du futur roi de Suède, Gustave III, encourage son jeune élève, alors âgé de 11 ans, à écrire des lettres, exercice qui rebute le prince:

Lisez les lettres de Madame de Sévigné; vous croirez entendre une conversation, une mère qui parle à sa fille comme si elles étaient vis-à-vis l'une de l'autre [...] On les [ces lettres] a louées, admirées, adoptées même pour modèles en fait de lettres, parce qu'elles étaient simples et naturelles. [...] Tout ce qui ressemble à la conversation est bon, tout ce qui a l'air plus apprêté sera infailliblement condamné par le bon goût. ${ }^{66}$

La compétence linguistique est clairement associée à l'esthétique épistolaire héritée du modèle culturel français que le jeune prince est censé s'approprier, ce qu'il fera à merveille aussi bien dans ses lettres à Marmontel qui suscitent le jugement admiratif de Diderot que dans celles écrites à son frère ou à sa mère. ${ }^{67}$

Outre la compétence linguistique, les parents désirent aussi inculquer les valeurs et les normes propres à leur rang social ou au rôle auxquels on les destine, comme on peut le voir aussi bien dans la correspondance

absents, elle doit naturellement être comme une conversation libre entre des amis présents, qui rarement est méthodique' [4 avril 1763] (cité dans Van der Vliet, Onno Zwiervan Haren, 286).

63 Cité dans Östman, 'French in Sweden', 293. 'Drogues' signifie ici 'choses sans valeur'.

64 Gretchanaia, 'Autobiographie des femmes russes', 133.

65 Ruberg, 'Children's correspondence', 299, note 14.

66 Cité dans Fumaroli, Quand l'Europe parlait français, 354.

67 Ibidem, 353, 355-359.

\section{Amsterdam University Press}


entre Marie-Antoinette et sa mère Marie-Thérèse d'Autriche ${ }^{68}$ qu'entre Van Hogendorp et sa mère Carolina ${ }^{69}$, ou encore entre Isabelle de Charrière et son neveu néerlandais. Mais dans ce cadre codé se fait jour l'expression des sentiments favorisée par la confidentialité créée dans l'intimité de la lettre, requise parfois par les parents..$^{70}$ Dans les échanges épistolaires s'étalant sur une longue période, se dessine aussi le développement d'une personnalité comme on peut le voir dans les lettres de Van Hogendorp ou dans celles de Marie-Antoinette à sa mère: 'l'ensemble de cette correspondance montre le passage d'une enfant maladroite à une jeune femme épanouie, [...] d'une Dauphine gauche et timide à la reine de France'.$^{1{ }^{1}}$

Linfluence des romans épistolaires français, à commencer par les Lettres portugaises (1669) de Guilleragues jusqu'à Julie ou la nouvelle Héloïse (1761) de Rousseau, qui ont eu un retentissement indéniable en Europe, a contribué à la promotion du français, langue de la relation amoureuse, on peut en citer divers exemples.

Ainsi, la correspondance en français de Nikolai Karamzin avec sa fiancée et seconde femme montre qu'il considère cette langue comme convenable à la communication intime et confidentielle - la connaissance de La Nouvelle Héloïse favorisant visiblement l'expression de ses sentiments..$^{72}$ Lirruption du français dans les lettres de Giustiniana Wynne à son amant italien Andrea $\mathrm{Memmo}^{73}$, dénote non seulement l'intégration de valeurs véhiculées par les salons et les discussions d'ordre culturel en France, mais aussi une technique de séduction épistolaire, chargée de tensions érotiques et émotionnelles. Dans la Roumanie du dix-neuvième siècle, le grand poète roumain, Mihaïl Eminescu (1850-1889), bien que ne possédant qu'imparfaitement le français, choisit délibérément cette langue pour s'adresser à sa bien-aimée, Véronica Micle, 'Madame je vous écris en français', la considérant comme propre à l'expression de ses sentiments amoureux. ${ }^{74}$ Enfin, au tout début du vingtième siècle, dans la Turquie de la fin de l'Empire ottoman, les carnets de Tevfik Riza Bey dédiés à sa jeune femme montrent l'impact de la littérature française, aussi bien Rousseau que les romantiques du dix-neuvième siècle, sur le registre du discours amoureux ${ }^{75}$.

68 Seth, Marie-Antoinette, 1-111.

$69 C f$. Van Strien-Chardonneau, dans ce volume.

70 Ruberg, 'Children's correspondence', 304.

71 Seth, Marie-Antoinette, 2.

72 Sapchenko, 'French and Russian in Ego-Documenten', 157-159.

Cf. Isenberg, dans ce volume.

Mihaila, 'The Beginnings and the Golden Age of Francophonie among the Romanians', 345 .

5 Cf. Güven \& Güven dans ce volume. 


\section{Langue de la réflexion intérieure et de la formation de la pensée}

Dans le troisième tiers du dix-huitième siècle, nous l'avons vu, l'adjectif intime qui désignait jusqu'alors une relation privilégiée avec autrui - se met aussi à impliquer un sentiment intérieur, une conscience propre au sujet, ${ }^{\prime}{ }^{6}$ et l'on voit se développer ce qui constitue l'intime au sens moderne du terme, l'expression des émotions et des sentiments, en particulier le sentiment de la nature, le questionnement existentiel sur le sens de la vie.

Bien que placée sous le signe de l'échange relationnel, la lettre peut offrir un espace à l'intériorité du sujet: ainsi Francesca Piselli montre, dans ce volume, comment l'écriture épistolaire de la comtesse d'Albany, tout en s'inscrivant dans la pratique salonnière de la conversation en français, favorise l'émergence d'un discours personnel, intime. La correspondance secrète, entretenue par Isabelle de Charrière dans sa jeunesse avec Constant d'Hermenches, prend assez souvent les allures d'un journal intime centré sur la personne de l'épistolière. C'est cependant le journal qui offre le plus souvent l'espace nécessaire à cette expression de l'intériorité du scripteur, même dans le cas du journal de voyage plus orienté sur le monde extérieur. Ainsi, 'l'analyse du journal de voyage de la comtesse Waleria Tarnowska par Michel Braud montre que le récit de voyage laisse souvent la place à l'épanchement intime'. ${ }^{77}$ C'est une évolution analogue que signale Martina Musilová dans les journaux personnels d'Alexandrine von Dietrichstein (1775-1847): ceux de 1797-1798 relatant soirées, voyages et rencontres sont essentiellement factuels tandis que celui de 1804 , rédigé dans un style où domine l'affectif, contient nombre de réflexions sur sa vie et ses sentiments. ${ }^{78}$

Le jeune Willem René van Tuyll van Serooskerken, dans le journal tenu lors de son séjour en Suisse, laisse parfois percer sa mélancolie et s'interroge sur le sens de sa vie:

Premier jour du $19^{\mathrm{e}}$ siècle. Qu'ai-je fait, qu'ai-je vu ce matin? J'ai baguenaudé, j'ai ôté des taches à mon habit et j'ai vu les gens de Colombier donner une mascarade. Voilà ce qui s'appelle bien commencer un siècle. Mais quelle raison aurait-on de croire que les hommes furent plus sages ou plutôt moins fous le $1^{\text {er }}$ janvier 1800 que le 31 décembre 1799 ? Aucune.

76 Montémont, 'Dans la jungle de l'intime', 18-38; elle y précise aussi que 'le Dictionnaire de l'Académie française mentionne 'intime' seulement dans la combinaison 'ami intime' dans l'édition de 1694 mais ajoute en 1798 la notion de 'persuasion intime'.

77 Gretchanaia \& Viollet, Si tu lis jamais ce journal ..., 18.

78 Musilová, 'Contes, romans et journaux inédits de la princesse Alexandrine von Drietrichstein', 165-166.

\section{Amsterdam University Press}


Ce sont les mêmes hommes et par conséquent les mêmes passions, les mêmes travers, les mêmes ridicules qui les gouvernent. Non je le répète, pour qu'on voie les hommes changer et devenir meilleurs, il faut que le bon Dieu en crée de nouveaux. ${ }^{79}$

Par contre, les lettres écrites à sa mère, et rédigées conjointement au journal, contiennent régulièrement des mots ou des phrases en néerlandais dans lesquelles s'exprime l'affectivité du jeune homme, très attaché à cette mère dont il est éloigné, et souffrant parfois du mal du pays. ${ }^{80}$ Le questionnement existentiel s'exprime en français, la relation d'intimité avec la mère également mais avec une résurgence récurrente de la langue maternelle.

L'influence déjà évoquée des Confessions de Rousseau a joué un grand rôle dans l'élaboration de cette écriture de l'intériorité. Gretchanaia signale que, si l'influence rousseauiste dans les écrits autobiographiques en français de femmes russes nées dans la première moitié du dix-huitième siècle est moindre que celle des Mémoires aristocratiques français, elle est plus sensible chez les femmes de la génération ultérieure. Elle cite ainsi l'exemple d'une femme russe, restée anonyme qui rédige en russe une Note secrète surma vie de 1794 à 1808 dans les années 1820 mais donne en conclusion une sorte de résumé en français qui commence par la réécriture du début des Confessions: la diariste s'approprie les objectifs de Rousseau ('J'ai dit le bien et le mal avec la même franchise de Rousseau. Je n'ai rien tu de mauvais, rien ajouté de bon') tout en les inscrivant dans sa condition de femme et son expérience propre. ${ }^{81}$

S'interrogeant sur les fonctions respectives du français et du russe dans les lettres de Karamzin, Sapchenko écrit: 'The functions of the two languages are noticeably differentiated in Karamzin's letters: French is the language of thoughts and feelings whereas Russian is the language of events, deeds and actions'. ${ }^{82}$

On trouve une opposition du même ordre chez Van Hogendorp dans la période où il se réapproprie le néerlandais ainsi que chez son frère aîné, Dirk. Il est possible que les débats sur les langues et le langage, illustrés, entre autres par Beauzée, Dumarsais, Condillac, et qui ont des répercussions sur les méthodes d'enseignement du français aux dix-huitième et dix-neuvième 
siècles, aient pu renforcer la prise de conscience du lien entre apprentissage de la langue française et formation de la pensée. ${ }^{83}$

Pour Isabelle de Charrière, par exemple, l'enseignement des langues étrangères est non seulement une acquisition linguistique mais encore une éducation à la pensée. Létude des langues, écrit-elle, et en particulier celle du français, est moins précieuse par le but qu'on atteint (la communication avec l'autre) que le chemin qui y mène, à savoir la traduction qui oblige à réfléchir au sens exact des mots, la maîtrise de la syntaxe, permettant, elle, d'éclaircir et préciser les idées. Et elle considère que le bilinguisme néerlandais/ français, courant dans son milieu d'origine, est l'occasion d'un exercice intellectuel en ce qu'il oblige à une comparaison entre les deux langues et donc à une réflexion sur le langage en général (O.C., V:284).

\section{Langue de la prise de conscience identitaire nationale}

Iouri Lotman a avancé que la politique d'occidentalisation plus ou moins forcée menée en Russie par Pierre le Grand a fait des nobles russes des étrangers dans leur propre pays, la pratique des Russes parlant français entre eux constituant un argument illustrant son hypothèse. ${ }^{84}$ Ce que Lotman définit pour tout un groupe social, la noblesse russe du dix-huitième siècle, semble avoir été vécu au niveau individuel par la jeune Belle de Zuylen (Isabelle de Charrière). Elle a en effet éprouvé ce sentiment d'étrangeté: ne confie-t-elle pas à Constant d'Hermenches en 1764: 'c'est en vérité une chose étonnante que je m'appelle Hollandaise et Tuyll' $(O . C$., I, 342) et plus tard en 1768, elle avouera: 'je voudrais être du pays de tout le monde' (O.C., II, 80). Les cercles littéraires qu'elle fréquente alors à Utrecht et dans lesquels le français est de rigueur, la langue française et sa littérature constituent une patrie spirituelle privilégiée dans un monde où elle se sent mal acceptée.

Cependant, comme l'a montré Michelle Lamarche Marrese, en s'appuyant sur les correspondances familiales de nobles russes du dix-huitième siècle et du début du dix-neuvième, le bilinguisme et le biculturalisme n'entraînent pas nécessairement le rejet de la langue et de la culture d'origine et elle remet en question le modèle lotmanien du Russe noble étranger dans son propre pays:

83 Hassler, 'Enseignement de la langue et formation de la pensée', 109-119.

84 Burke, 'Diglossia in Modern Europe', 48-49.

\section{Amsterdam University Press}


Unlike Lotman, who emphasized the 'consciously theatrical' behavior of the Europeanized nobility and only minimally addressed the experience of women, I maintain that the worldview of much of the Russian elite was characterized by unproblematic cultural bilingualism. Familiarity with Western and Russian ways of life permitted nobles of both sexes to participate to varying degrees in European culture without detracting from their feelings of belonging to Russia and to experience both forms of behavior as natural. ${ }^{85}$

Le cas de Valerii Levashov (1822-1877) semblerait de prime abord pouvoir illustrer la thèse de Lotman. L'utilisation du français dans sa correspondance permet de préserver une habitude - le français cohabitant avec le russe et d'autres langues - et de maintenir et affirmer une distinction sociale. Mais c'est surtout une langue refuge qui le protège de l'ignoble monde réel, sans pour autant le couper de son peuple, en particulier de ses paysans dont il s'efforce d'améliorer le sort. ${ }^{86}$

Le français joue ce même rôle de langue refuge pour le futur Frédéric II et sa sœur la Margrave de Bayreuth qui s'écrivent en français. Langue de leur éducation par une gouvernante et un précepteur français auxquels ils étaient fort attachés, elle offre au frère et à la sœur dont l'enfance fut saccagée par un père odieux, un espace chaleureux et protecteur, propice à la confidence et à une commune espérance en des jours meilleurs, sans par ailleurs leur faire oublier qu'ils sont avant tout 'des princes Hohenzollern, attachés, becs et ongles, aux intérêts de leur maison'. ${ }^{87}$ Et tout francophile fût-il, Frédéric II a perçu l'essor de la langue et de la culture allemandes. ${ }^{88}$

C'est en effet, comme l'a constaté Peter Burke, à partir du milieu du dix-huitième siècle, que s'établit dans divers pays européens un lien de plus en plus étroit entre langage et nation. Burke souligne l'importance des penseurs allemands tels que Herder, Fichte, Schlegel dans la diffusion de l'idée qu'une nation est une communauté liée par la langue. ${ }^{89}$

Sur ce point, on constate l'évolution d'Isabelle de Charrière qui se plaît, vers la fin de sa vie, à intercaler de temps à autre quelques mots ou phrases en néerlandais dans les lettres à sa famille et qui surtout conseille

85 Lamarche Marrese, “The Poetics of Everyday Behavior” Revisited', 705.

86 'Family correspondence in the Russian nobility: a letter of 1847 from Valerii Levashov to his cousin, Ivan D. Iakushkin'. [En ligne: https://frinru.ilrt.bris.ac.uk/introduction/ family-correspondence-russian-nobility-letter-1847-valerii-levashov-his-cousin-ivan-d].

87 Fumaroli, Quand l'Europe parlait français, 135-136.

88 Böhm, 'The Domains of Francophonie and Language Ideology', 183-185.

89 Burke, Language and Communities in Early Modern Europe, 163-164.

\section{Amsterdam University Press}


à son neveu une prise de distance avec le modèle de sociabilité française, incompatible, à ses yeux, avec le caractère national hollandais. Mais ceci n'implique nullement le rejet du bilinguisme et elle insiste conjointement sur la nécessité et de maîtriser le français et de posséder parfaitement le néerlandais. C'est d'ailleurs ce qu'elle avait déjà recommandé en 1785 à la mère de l'enfant: 'Enseignez-lui donc vous-même avec soin le hollandais, qu'il le lise, le parle, l'écrive correctement et avec grâce, que le français ne gâte point son hollandais, qu'il ne le mêle point, qu'il ne francise point sa propre langue' (O. C., II, 459).

Rentrant en Hollande après un long séjour à Berlin, le jeune Van Hogendorp, craignant d'être un étranger dans sa patrie, affirme son désir de réapprendre le néerlandais et de se 'renationaliser'. La réappréciation de sa langue originelle s'accompagne d'une critique du modèle culturel français et d'une prise de conscience du 'génie' peu compatible des langues française et néerlandaise. C'est en français néanmoins qu'il exprime cette prise de conscience, et c'est en français qu'il continuera à écrire lettres et journaux personnels.

Dans différents pays et en des périodes variées pré-révolutionnaires, révolutionnaires ou coloniales, le questionnement identitaire a été stimulé par la pratique d'une langue allogène et la connaissance de la culture qui y est liée, culture de l'autre souvent fantasmée. C'est par elles que prennent forme la prise de conscience identitaire et la recherche d'appartenance nationale, que ce soit dans les Pays-Bas du dix-huitième siècle ou dans la Turquie encore ottomane de İzzet Melij Devrim qui, dans Leïla, œuvre écrite en français, revendique indirectement son identité ottomane et se la réapproprie par le biais de son personnage.

\section{Bibliographie}

Baggerman, Arianne \& RudolfDekker, Child of the Enlightenment, Revolutionary Europe reflected in a Boyhood Diary (Leiden: Brill, 2009).

Baggerman, Arianne, Rudolf Dekker \& Michael Mascuch (eds.), Controlling Time and Shaping the Self (Leiden: Brill, 2011).

Bandelier, André, 'Échanges épistolaires et préceptorat des Lumières' Documents pour l'histoire du français langue étrangère ou seconde [SIHFLES, 2002], 29, 145-173.

Beaufort, Henriette L.T. de, Gijsbert Karelvan Hogendorp, grondleggervan het Koninkrijk, $4{ }^{\mathrm{e}}$ éd. (Rotterdam: Donker, 1979).

Böhm, Manuela, 'The Domains of Francophonie and Language Ideology in Eighteenth- and Nineteenth-Century Prussia', in European Francophonie, ed. by Rjéoutski, Argent \& Offord, 178-207.

\section{Amsterdam University Press}


Bourdieu, Pierre, La distinction: critique sociale du jugement (Paris: Éditions de Minuit, 1979).

Brunot, Ferdinand, Histoire de la langue française des origines à $19 o o$ (Paris: Armand Colin, 1905-1953, 1969-1979), 13 vol.

Burke, Peter, 'Diglossia in Early Modern Europe', in European Francophonie, ed. by Rjéoutski, Argent \& Offord, 33-49.

Burke, Peter, Language and Communities in Early Modern Europe (Cambridge: Cambridge University Press, 2004).

Cerman, Ivo, 'Aristocratic French Literature in Bohemia', in European Francophonie, ed. by Rjéoutski, Argent \& Offord, 209-238.

Charrière, Isabelle de, CEuvres complètes [O.C.] (Amsterdam: G.A. van Oorschot, 1979-1984), 10 vol. Chevalier, Jean-Claude, 'L'Histoire de la langue française de Ferdinand Brunot', in Pierre Nora (éd.), Lieux de mémoire III. Les France, 2. Traditions, 421-459 (Paris: Gallimard, 1992).

Chevalier, Jean-Claude, 'Ferdinand Brunot et le français langue étrangère', Documents pour l'histoire du français langue étrangère ou seconde [SIHFLES, 1989], 3, 12-14.

Cots Vicente, Montserrat, compte rendu de Benoît Melançon (éd.), L'Invention de l'intimité au siècle des Lumières. Littérales, 17. Nanterre, Université Paris X-Nanterre, 1995, Eighteenth-Century Fiction, 9, (1997/2), 242-243.

Coudreuse, Anne \& Françoise Simonet-Tenant (éds), Pour une histoire de l'intime et de ses variations, (Paris: L'Harmattan, 2009).

Dufief, Pierre-Jean, 'Introduction', in Les écritures de l'intime. La correspondance et le journal. Actes du colloque de Brest 23-24-25 Octobre 1997. Textes rassemblés et présentés par PierreJean Dufief, (Paris: Honoré Champion, 2000).

Dulac, Georges, 'The Use of French by Catherine II in her Letters to Friedrich Melchior Grimm (1774-1796)', in French and Russian in Imperial Russia, ed. by Offord, Ryazanova-Clarke, Rjéoutski \& Argent, 1, 45-6o.

Frijhoff, Willem \& André Reboullet (éds), Le Français dans le monde. Recherches et Applications. Histoire de la diffusion et de l'enseignement du français dans le monde (Paris: Hachette, janvier 1998).

Fumaroli, Marc, Quand l'Europe parlait français (Paris: de Fallois, 2001).

Gretchanaia Elena, 'Les écrits autobiographiques des femmes russes du XVIII ${ }^{\mathrm{e}}$ siècle rédigés en français', Dix-huitième siècle, 36 (2004), 131-154.

Gretchanaia, Elena \& Catherine Viollet, Situ lis jamais ce journal... Diaristes russes francophones 1780-1854 (Paris: CNRS Éditions, 2008).

Gretchanaia, Elena, Alexandre Stroev \& Catherine Viollet (éds), La francophonie européenne aux XVIII ${ }^{e}$-XIX $X^{e}$ siècles. Perspectives littéraires, historiques et culturelles (Bruxelles/Bern/ Berlin: Peter Lang, 2012).

Gusdorf, Georges, Les écritures du moi (Paris: Éditions Odile Jacob, 1991).

Hassler, Gerda, 'Enseignement de la langue et formation de la pensée: un objectif idéologique de l'enseignement du français au XVIII ${ }^{\mathrm{e}}$ et début du XIX ${ }^{\mathrm{e}}$ siècle', Documents pour l'histoire du français langue étrangère ou seconde [SIHFLES, 1991], 8, 109-119.

Kok Escalle, Marie-Christine \& Madeleine van Strien-Chardonneau, 'Moyens linguistiques et construction identitaire sexuée dans l'enseignement de la langue française aux Pays-Bas XVII ${ }^{\mathrm{e}}$-XVIII ${ }^{\mathrm{e}}$ siècles', Beiträge zur Geschichte der Sprachwissenschaft, 21 (2011), 219-238.

Kok Escalle, Marie-Christine, Nadia Minerva \& Marcus Reinfried (éds), 'Histoire internationale del'enseignement du français langue étrangère ou seconde: problèmes, bilans et perspectives'. Recherches et Applications 52. Le français dans le monde (Paris: Clé international, juillet 2012). 
Lamarche Marrese, Michelle, 'Princes Dashkova and the Politics of Language in Eigtheenth-Century Russia', in French and Russian in Imperial Russia, ed. by Offord, Ryazanova-Clarke, Rjéoutski and Argent, 2, 31-47.

Lamarche Marrese, Michelle, “The Poetics of Everyday Behavior” Revisited. Lotman, Gender, and the Evolution of Russian Noble Identity', Kritika: Explorations in Russian and Eurasian History 11, 4 (2010), 701-739.

Lejeune, Philippe, Conférence aux Journées de l'Autobiographie (APA), Rousseau et la révolution autobiographique. Genève, 26 mai 2012 [En ligne: http://www.autopacte.org/Rousseau.pdf]).

Lejeune, Philippe, 'les journaux spirituels en France du XVI ${ }^{\mathrm{e}}$ au XVII ${ }^{\mathrm{e}}$ siècle', Littérales, 33 (2004), Problématiques de l'autobiographie, 63-85.

Mihaila, Ileana, 'The Beginnings and the Golden Age of Francophonie among the Romanians', in European Francophonie, ed. by Rjéoutski, Argent \& Offord, 337-370.

Minerva, Nadia, 'De Ferdinand Brunot à la SIHFLES. Pour une histoire de la recherche', Le Français dans le monde. Recherches et Applications. Histoire de la diffusion et de l'enseignement du français dans le monde (Paris: Hachette EDICEF, 1998), 180-186.

Minerva, Nadia \& Marcus Reinfried, 'Les domaines à explorer et l'évolution historique', 'Histoire internationale de l'enseignement du français langue étrangère ou seconde: problèmes, bilans et perspectives', Recherches et Applications. Le français dans le monde (Paris: CLE International, 2012), 14-28.

Montémont, Véronique, 'Dans la jungle de l'intime: enquête lexicographique et lexicométrique (16o6-2008)', in Coudreuse \& Simonet-Tenant (éds), Pour une histoire de l'intime et de ses variations, (Paris: L'Harmattan, 2009), 15-38. [En ligne: https://itineraires.revues.org/585].

Murphy, Emilie, 'Récits de voyage rédigés en français par des femmes russes (1777-1850)', in Gretchanaia, Stroev \& Viollet (éds), La francophonie européenne aux XVIII ${ }^{e}$-XIX $X^{e}$ siècles, 221-235.

Musilová, Martina, 'Contes, romans et journaux inédits de la princesse Alexandrine von Dietrichstein, née comtesse Chouvalova', in Gretchanaia, Stroev \& Viollet (éds), La Francophonie européenne aux XVIII'-XIX ${ }^{e}$ siècles, 159-171.

Offord, Derek, Lara Ryazanova-Clarke, Vladislav Rjéoutski \& Gesine Argent (eds.), French and Russian in Imperial Russia, 2 vol., 1. Language use among the Russian Elite, 2. Languages attitudes and identity (Edinburg: Edinburgh University Press, 2015).

Östman, Margaret, 'French in Sweden in the Seventeenth, Eighteenth and Nineteenth Centuries', in European Francophonie, ed. by Rjéoutski, Argent \& Offord, 173-306.

Rey, Alain, Frédéric Duval \& Gilles Siouff, Mille ans de langue française, histoire d'une passion, (Paris: Perrin / Tempus, 2007, éd. revue et augmentée, 2011), 2 vol.

Rjéoutski, Vladislav, Gesine Argent \& Derek Offord (eds.), European Francophonie. The Social, Political and Cultural History of an International Language (Oxford/Bern/Berlin: Peter Lang, 2014).

Rjéoutski, Vladislav \& Alexandre Tchoudinov (éds), Le précepteur francophone en Europe (XVII ${ }^{e}$ $X I X^{e}$ siècles) (Paris: L'Harmattan, 2013).

Ruberg, Willemijn, 'Children's correspondence as a pedagogical tool in the Netherlands (17701850)', Pedagogica Historica: International Journal of the History of Education, 41:3, 295-312, [En ligne http://dx.doi.org/10.108o/o0309230500069779].

Ruberg, Willemijn, 'Letter Writing and Elite Identity in the Netherlands, 1770-1850', Scandinavian Journal of History, 30, 2005 (3-4), 249-258, [En ligne http://dx.doi. org/10.1080/03468750500272579].

Sapchenko, Liubov, 'French and Russian in Ego-Documenten by Nikolai Karamzin', in French and Russian in Imperial Russia, ed. by Offord, Ryazanova-Clarke, Rjéoutski \& Argent, 1, 152-171.

\section{Amsterdam University Press}


Seth, Catriona, Marie-Antoinette. Anthologie et dictionnaire (Paris: Robert Laffont, 2006).

Simonet-Tenant, Françoise, 'À la recherche des prémices d'une culture de l'intime', Itinéraires, 4 (2009), 39-62. [En ligne https://itineraires.revues.org/1466].

Siouffi, Gilles, 'De l"universalité' européenne du français au XVIII siècle: retour sur les représentations et les réalités', Langue française, 3, (2010), 13-29.

Strien-Chardonneau, Madeleine van, 'Willem René van Tuyll van Serooskerken en visite à Colombier (mai 1799-mars 1800). Documents inédits', Cahiers Isabelle de Charrière/Belle de Zuylen Papers, 5 (2010), 75-120.

\section{About the authors}

Madeleine van Strien-Chardonneau is a retired lecturer in French language and culture and an Associate Member of LUCAS (Leiden University Centre for the Arts in Society). Her scholarly interests include the accounts of French travelers in Holland in the eighteenth and nineteenth centuries, the history of the teaching of French in Holland, the francophone Dutch writer Isabelle de Charrière and the personal writings of Netherlanders in French in the eighteenth and early nineteenth centuries.

Email: m.m.g.van.strien-chardonneau@hum.leidenuniv.nl

Marie-Christine Kok Escalle, Associate Professor in French cultural history and Intercultural Communication at Utrecht University until her recent retirement, is a member of the Institute for Cultural Inquiry (Utrecht University). Her scholarly interests include the cultural role the French language has played in the Netherlands, specially in the eighteenth and nineteenth centuries, and the development of intercultural competence through foreign language learning and teaching in the past as well as nowadays. She is also a longstanding member of the SIHFLES.

Email: M.C.J.Kok-Escalle@uu.nl 


\section{Part I}

From French as a Language of the Bilingual Netherlands to the 'Language of Universality' in a Wider Europe (Sixteenth - Eighteenth Centuries)

Du français langue du bilinguisme aux Pays-Bas à la 'langue de l'universalité' dans l'Europe élargie (seizième - dix-huitième siècles) 
Amsterdam University Press 